\title{
Weak gravitational lensing by Einstein-non-linear-Maxwell-Yukawa black hole
}

\author{
Wajiha Javed, ${ }^{1, *}$ Muhammad Bilal Khadim, ${ }^{1, \dagger}$ and Ali Övgün ${ }^{2,3, \ddagger}$ \\ ${ }^{1}$ Division of Science and Technology, University of Education, Township-Lahore, Pakistan \\ ${ }^{2}$ Instituto de Física, Pontificia Universidad Católica de Valparaíso, Casilla 4950, Valparaíso, Chile. \\ ${ }^{3}$ Physics Department, Arts and Sciences Faculty, Eastern Mediterranean University, Famagusta, North Cyprus via Mersin 10, Turkey.
}

(Dated: January 12, 2020)

\begin{abstract}
In this article, we analyze the weak gravitational lensing in the context of Einstein-non-linear Maxwell-Yukawa black hole. To this desire, we derive the deflection angle of light by Einstein-nonlinear Maxwell-Yukawa black hole using the Gibbons and Werner method. For this purpose, we obtain the Gaussian optical curvature and implement the Gauss-Bonnet theorem to investigate the deflection angle of Einstein-non-linear Maxwell-Yukawa black hole. Moreover, we derive the deflection angle of light in the presence of plasma medium. We also analyze the graphical behavior of deflection angle by Einstein-non-linear Maxwell-Yukawa black hole in the presence of plasma as well as non-plasma medium.
\end{abstract}

PACS numbers: 95.30.Sf, 98.62.Sb, 97.60.Lf

Keywords: Weak gravitational lensing; Einstein-non-linear Maxwell-Yukawa black holes; Deflection angle; Gauss-Bonnet theorem.

\section{INTRODUCTION}

In our universe the black holes are essential components and when stars die, they collapse and become very small objects which is the most important discoveries of astrophysics. To test the fundamental laws of universe black holes provide a golden opportunity. For example neutron star merges and the gravitational waves from black holes has been discover recently. Therefore, the gravitational lensing by black holes has attain incredible attention in the last few decade, essentially because of the solid proof about the presence of supermassive black holes at the focal point of galaxies [1,2]. By utilizing the gravitational lensing we can simplified the study of black holes, which is a general investigative method for acquiring the time delays of the images, magnification and positions. Erasmus Darwin [3] was the first who introduced the schwarzschild geometry. After very soon in 1985 Walliam Herschel [4] published his similar article and after that many authors [5, 6], extended this geometry to general spherically symmetric black holes and Reissner-Nordstrom geometry. Kerr black holes [7]-[10] was also discussed for acquiring the time delays of the images, magnification and positions by using gravitational lensing. Many modification has been done through deflection of light and modification in the reference of non-linear electrodynamics (NLE) [11] has been studied through the various alternative gravity theories [12]. Classically, a black hole contains singularity and also horizon because in general theory of relativity the specetime singularities create a dozen of problems which are physical as well as mathematical. Therefore many people use various methods to remove these singularities from the black holes such as modified gravity, effect of quantum gravity and NLE. Bozza [13, 14] discussed these topics in his recent article in details which also include observational prospectus and additional references [15]-[67].

The main focus of this article is to calculate the deflection angle of photon by using GBT. The aspect of deflection of light has been widely studied in different astrophysical system in the influence of strong as well as weak gravitational lensing [20]-[24]. Gibbons and Werner [25] made a very important role who contended about the significance of global topology on the deflection of light by utilizing the optical geometry and GBT. Additionally, they have calculated deflection angle for the schwarzschild black holes which is different from the other method by supposing domain outside of the light ray, where the mass is closed in the given area on space is strongly related to the lensing effect. Recently, Gibbons and Werner (GW) [25] computed the deflection angle by applying GBT as follows

$$
\alpha=-\iint_{D \infty} \mathcal{K} d S .
$$

\footnotetext{
*Electronic address: wajiha.javed@ue.edu.pk; wajihajaved84@yahoo.com

${ }^{\dagger}$ Electronic address: blaljutt723@gmail.com

‡Electronic address: ali.ovgun@pucv.cl; ali.ovgun@emu.edu.tr; URL: https : / / a ovgun.weebly.com
} 
Here, $\mathcal{K}$ is Gaussian curvature and $d S$ represents the surface element. Afterwards, Werner [26],[27] extend this method and obtained the deflection of light by Kerr-Newman BH by applying the Nazims's method for RanderFinsler metric. Recently, deflection angle for a static spherically symmetric and asymptotically flat spacetime by using the finite distance from an observer to a light source has been calculated by Ishihara et al. [28]-[31]. Moreover, Asada et al. [32]in stationary axisymmetric spacetime have calculated the weak gravitational lensing. In all of these methods by using GBT they have calculated the weak gravitational lensing which shows global properties. The study of gravitational lensing in the presence of plasma medium discussed in number of cases. Initially, Bisnovatyikogan and Latimer [33]-[34] show that the gravitational deflection in plasma is different from vacuum deflection angle due to presive properties of plasma. Recently, Gallo and Crisnejo [35] have studied the deflection angle of photon in a plasma medium.

Weak gravitational lensing for hairy BH in the back burner of Einstein-Maxwell theory (EMT) with a nonminimally coupled dilaton has been examined by Javed et al. [36]. After that, there is a lot of literature [37]-[78] related to the investigation of weak gravitational lensing through the method of GBT on various black holes, cosmic strings and wormholes. In this paper, we study the weak gravitational lensing by Einstein-non-linear MaxwellYukawa black holes.

This paper is composed as follows; In Section 2 We concisely review about Einstein-non-linear Maxwell-Yukawa black hole. In Section 3, By using the Gauss-Bonnet theorem we compute the deflection angle of Einstein-non-linear Maxwell-Yukawa black hole. In Section 4, we enhance our work to investigate the deflection angle in the influence of plasma medium and in addition we demonstrate the graphical impact of deflection angle in the context of Einsteinnon-linear Maxwell-Yukawa black hole for plasma as well as non-plasma medium. In Section 5, we recap our results which we obtain in present work.

\section{COMPUTATION OF WEAK LENSING BY EINSTEIN-NON-LINEAR MAXWELL-YUKAWA BLACK HOLE AND GAUSS-BONNET THEOREM}

The Einstein-non-linear Maxwell-Yukawa BH in a static and spherically symmetric form is given as [79]

$$
d s^{2}=-f(r) d t^{2}+\frac{d r^{2}}{f(r)}+r^{2}\left(d \theta^{2}+\sin ^{2} \theta d \phi^{2}\right)
$$

The metric function $f(r)$ yields

$$
f(r) \simeq 1+\frac{2 M}{r}-\frac{4 q C_{0}}{r^{2}}+\frac{4 q C_{0} \alpha}{3 r}-q C_{0} \alpha^{2}+\mathcal{O}\left(\alpha^{3}\right) .
$$

Here, $M$ rendered as mass of $\mathrm{BH}, C_{0}$ is an integration constant [80], $q$ represents charge of $\mathrm{BH}$ that is located at the origin and $\alpha$ is a positive constant and it can be chosen as $\alpha=1$.

The optical spacetime simply written in equatorial plane $\left(\theta=\frac{\pi}{2}\right)$ to get null geodesics $\left(d s^{2}=0\right)$

$$
d t^{2}=\frac{d r^{2}}{f(r)^{2}}+\frac{r^{2} d \phi^{2}}{f(r)}
$$

From Eq. 1 we obtain non-zero Christopher symbols given below

$$
\Gamma_{00}^{0}=\frac{-f^{\prime}(r)}{f(r)}, \Gamma_{11}^{0}=\frac{2 f(r)-r f^{\prime}(r)}{2 r f(r)}, \Gamma_{01}^{1}=\frac{r^{2} f^{\prime}(r)-f(r) 2 r}{2} .
$$

Now, we calculate the Ricci Scalar corresponds to the optical metric by using the non-zero Christopher symbols that are stated as:

$$
\mathcal{R}=\frac{-f^{\prime}(r) 2}{2}+f^{\prime \prime}(r) f(r)
$$

The Gaussian optical curvature that is evaluated as follows:

$$
\mathcal{K}=\frac{\text { RicciScalar }}{2}
$$


After simplifying, Gaussian optical curvature for Einstein-non-linear Maxwell-Yukawa black hole is stated as:

$$
\mathcal{K}=\frac{-f^{\prime}(r)^{2}}{4}+\frac{f(r) f^{\prime \prime}(r)}{2}
$$

where $f(r)$ is given in Eq. 2 so that Gaussian optical curvature for Einstein-non-linear Maxwell-Yukawa black hole in leading order term is obtained as;

$$
\mathcal{K} \approx-12 \frac{C_{0} q}{r^{4}}+4 \frac{C_{0} q \alpha}{3 r^{3}}+\left(2 r^{-3}-24 \frac{C_{0} q}{r^{5}}+4 \frac{C_{0} q \alpha}{r^{4}}\right) M
$$

\section{DEFLECTION ANGLE OF EINSTEIN-NON-LINEAR MAXWELL-YUKAWA BLACK HOLES AND GAUSS-BONNET THEOREM}

Now, by utilizing GBT, we evaluate the deflection angle of photon by Einstein-non-linear Maxwell-Yukawa BH. By using GBT in the region $\mathcal{G}_{R}$, given as

$$
\int_{\mathcal{G}_{R}} \mathcal{K} d S+\oint_{\partial \mathcal{G}_{R}} k d t+\sum_{t} \hat{\alpha}=2 \pi \mathcal{X}\left(\mathcal{G}_{R}\right)
$$

where, $k$ represent the geodesic curvature, and $\mathcal{K}$ denotes the Gaussian optical curvature and one can define $k$ as $k=\bar{g}\left(\nabla_{\hat{\alpha}} \hat{\alpha}, \check{\alpha}\right)$ in such a way that $\bar{g}(\hat{\alpha}, \hat{\alpha})=1$, where $\hat{\alpha}$ represent the unit acceleration vector and $\alpha_{t}$ denotes the exterior angle at $t^{t h}$ vertex respectively. As $R \rightarrow \infty$, we obtain the jump angles equal to $\pi / 2$, hence $\alpha_{O}+\alpha_{S} \rightarrow \pi$. Here, $\mathcal{X}\left(\mathcal{G}_{R}\right)=1$ is a Euler characteristic number and $\mathcal{G}_{R}$ denotes the non-singular domain. Therefore, we obtain

$$
\iint_{\mathcal{G}_{R}} \mathcal{K} d S+\oint_{\partial \mathcal{G}_{R}} k d t+\hat{\alpha}=2 \pi \mathcal{X}\left(\mathcal{G}_{R}\right) .
$$

where, the total jump angle is $\hat{\alpha}=\pi$, When $R \rightarrow \infty$, then the remaining part is $k\left(D_{R}\right)=\left|\nabla_{\dot{D}_{R}} \dot{D}_{R}\right| \cdot$ Considering the radial component for geodesic curvature that is described as:

$$
\left(\nabla_{\dot{D}_{R}} \dot{D}_{R}\right)^{r}=\dot{D}_{R}^{\phi} \partial_{\phi} \dot{D}_{R}^{r}+\Gamma_{\phi \phi}^{r}\left(\dot{D}_{R}^{\phi}\right)^{2}
$$

At very high $R, D_{R}:=r(\phi)=R=$ const. Thus, the leading term of equation Eq. 11 vanishes and $\left(\dot{D}_{R}^{\phi}\right)^{2}=\frac{1}{f^{2}\left(r^{\star}\right)}$. Recalling $\Gamma_{\phi \phi}^{r}=\frac{2 f(r)-r f^{\prime}(r)}{2 r f(r)}$, we get

$$
\left(\nabla_{\dot{D}_{R}^{r}} \dot{D}_{R}^{r}\right)^{r} \rightarrow \frac{-1}{R}
$$

and which proves that the geodesic curvature is not effected to the topological defects (i.e., $\left.k\left(D_{R}\right) \rightarrow R^{-1}\right)$. We can write $d t=R d \phi$. Thus;

$$
k\left(D_{R}\right) d t=\frac{1}{R} R d \phi
$$

From the pervious results, we get

$$
\iint_{\mathcal{G}_{R}} \mathcal{K} d s+\oint_{\partial \mathcal{G}_{R}} k d t={ }^{R \rightarrow \infty} \iint_{S_{\infty}} \mathcal{K} d S+\int_{0}^{\pi+\alpha} d \phi .
$$

At $0^{\text {th }}$ order weak field deflection limit of the light is defined as $r(t)=b / \sin \phi$. Therefore, the deflection angle stated as:

$$
\alpha=-\int_{0}^{\pi} \int_{b / \sin \phi}^{\infty} \mathcal{K} \sqrt{\operatorname{det} \bar{g}} d u d \phi
$$

here we put the leading term of Eq. 8 into above Eq. 15, so the obtained deflection angle upto leading order term is computed as:

$$
\alpha \simeq \frac{4 M}{b}+\frac{C_{0} M \alpha q \pi}{b^{2}}+8 \frac{C_{0} q \alpha}{3 b}-3 \frac{C_{0} q \pi}{b^{2}}-\frac{32 C_{0} M q}{3 b^{3}}
$$




\section{WEAK LENSING BY EINSTEIN-NON-LINEAR MAXWELL-YUKAWA BLACK HOLE IN A PLASMA MEDIUM}

This portion is based on the investigation of weak gravitational lensing by Einstein-non-linear Maxwell-Yukawa black hole in plasma medium. For Einstein-non-linear Maxwell-Yukawa black hole the refractive index $n(r)$, is obtain as,

$$
n(r)=\sqrt{1-\frac{\omega_{e}^{2}}{\omega_{\infty}^{2}} f(r)}
$$

where $\omega_{e}$ and $\omega_{\infty}$ are electron plasma frequency and photon frequency measured by an observer at infinity respectively, then the corresponding optical metric illustrated as

$$
d t^{2}=g_{i j}^{o p t} d x^{i} d x^{j}=n^{2}(r)\left[-f(r) d t^{2}+\frac{d r^{2}}{f(r)}+r^{2}\left(d \theta^{2}\right)\right] .
$$

The metric function $f(r)$ is defined in Eq. 2.4 and the non-zero christopher symbols corresponding to the optical metric are computed as:

$$
\begin{aligned}
& \Gamma_{00}^{0}=\left(1+\frac{\omega_{e}^{2} f(r)}{\omega_{\infty}^{2}}\right)\left[\frac{-\omega_{e}^{2} f^{\prime}(r)}{2 \omega_{\infty}^{2}}\right]-\frac{-f^{\prime}(r)}{f(r)}, \\
& \Gamma_{01}^{1}=\left(1+\frac{\omega_{e}^{2} f(r)}{\omega_{\infty}^{2}}\right)\left(\frac{-\omega_{e}^{2} f^{\prime}(r)}{2 \omega_{\infty}^{2}}\right)+\frac{2 f(r)-r f^{\prime}(r)}{2 r f(r)}, \\
& \Gamma_{11}^{0}=\left(1+\frac{\omega_{e}^{2} f(r)}{\omega_{\infty}^{2}}\right)\left(\frac{\omega_{e}^{2} f(r) f^{\prime}(r) r^{2}}{2 \omega_{\infty}^{2}}\right)+\frac{r^{2} f^{\prime}(r)-f(r) 2 r}{2}
\end{aligned}
$$

Now, by using the above non-zero christopher symbols the value of Gaussian optical curvature is found as:

$$
\begin{array}{r}
\mathcal{K} \approx 3 \frac{\omega_{e}^{2} M}{r^{3} \omega_{\infty}^{2}}+2 \frac{M}{r^{3}}-104 \frac{C_{0} q M \omega_{e}^{2}}{\omega_{\infty}^{2} r^{5}}-24 \frac{C_{0} q M}{r^{5}}-20 \frac{C 0 q \omega_{e}^{2}}{\omega_{\infty}^{2} r^{4}}-12 \frac{C_{0} q}{r^{4}}+16 \frac{q \alpha C_{0} \omega_{e}^{2} M}{\omega_{\infty}^{2} r^{4}}+4 \frac{q \alpha C_{0} M}{r^{4}} \\
+2 \frac{q \alpha C 0 \omega_{e}^{2}}{r^{3} \omega_{\infty}^{2}}+4 / 3 \frac{q \alpha C_{0}}{r^{3}}
\end{array}
$$

For this, we use GBT to compute the deflection angle in order to relate it with non-plasma. As follows, for calculating angle in the weak field area, as the light beams become a straight line. Therefore, the condition at zero order is $r=\frac{b}{\sin \phi}$.

$$
\alpha=-\lim _{R \rightarrow 0} \int_{0}^{\pi} \int_{\frac{b}{\sin \phi}}^{R} \mathcal{K} d S
$$

So, the deflection angle in the presence of plasma medium is defined as;

$$
\begin{array}{r}
\alpha \simeq \frac{4 m}{b}+4 \frac{q \alpha C_{0} M \omega_{e}^{2} \pi}{b^{2} \omega_{\infty}^{2}}+\frac{C 0 q M \alpha \pi}{b^{2}}+4 \frac{C_{0} q \alpha \omega_{e}^{2}}{b \omega_{\infty}^{2}}+8 / 3 \frac{q C_{0} \alpha}{b}-5 \frac{C_{0} q \omega_{e}^{2} \pi}{b^{2} \omega_{\infty}^{2}}-3 \frac{q C_{0} \pi}{b^{2}}-\frac{416 q C_{0} M \omega_{e}^{2}}{9 b^{3} \omega_{\infty}^{2}} \\
-\frac{32 C_{0} q M}{3 b^{3}}+6 \frac{M \omega_{e}^{2}}{b \omega_{\infty}^{2}}
\end{array}
$$

The above result tells us that photon rays are moving into medium of homogeneous plasma. One can see that the plasma effect can be removed by neglecting $\frac{\omega_{e}}{\omega_{\infty}}$ term from Eq. 22 and it reduced into Eq. 16.

\section{GRAPHICAL INFLUENCE OF DEFLECTION ANGLE ON EINSTEIN-NON-LINEAR MAXWELL-YUKAWA BLACK HOLE}

This portion of paper comprises the graphical influence of deflection angle on Einstein-non-linear MaxwellYukawa BH. We examine the impact of different parameters on deflection angle. Here for simplicity we take $C \circ=C$ 

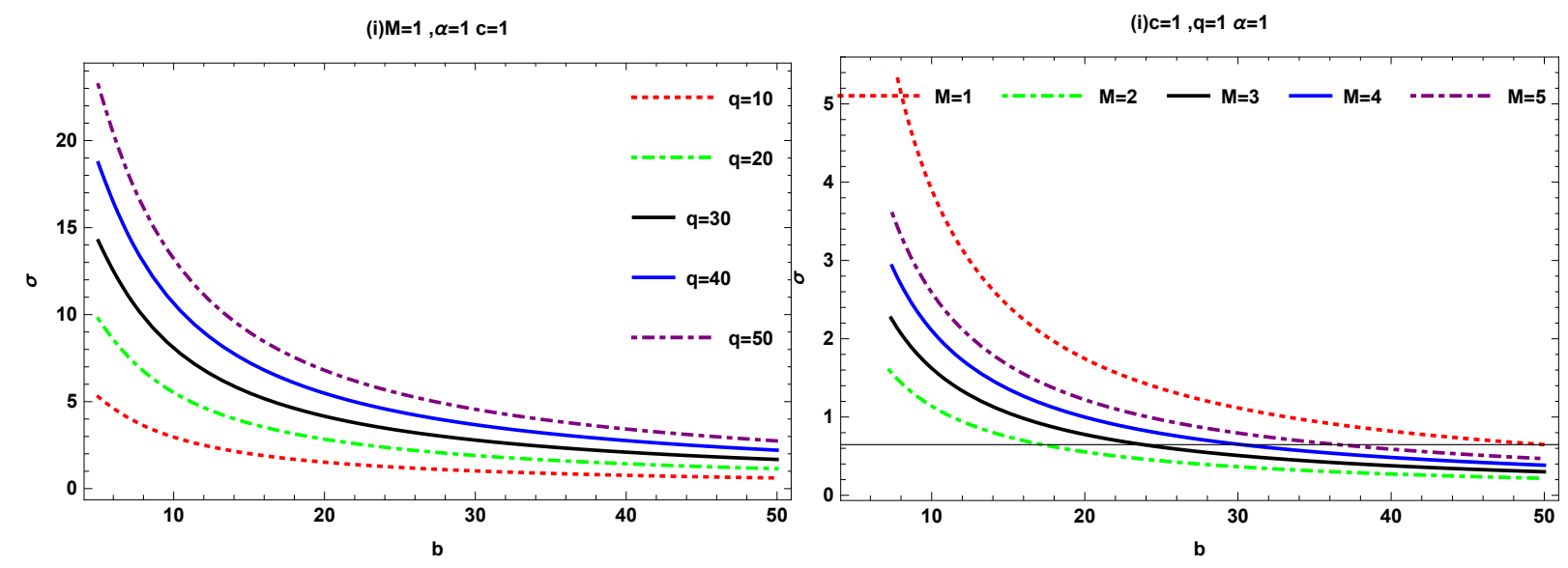

Figure 1: $\alpha$ versus $b$.

- Figure 1 indicates the behavior of deflection angle w.r.t $b$ by fixing the value of $M, c, \alpha$ and varying $q$. It is to be observed that for small constant value of $M$ and $q \geq 0$ the behavior of deflection angle gradually decreasing. For values $q<0$ we noticed that the deflection angle gradually negatively increasing. For large value of $M$ and $q \geq 0$ the deflection angle decreasing gradually.

- Figure 2 represents the behavior of deflection angle w.r.t $b$ by varying the mass $M$ and taking $q, c, \alpha$ fixed. We noticed that for values of $M \geq 0$ the behavior of deflection angle gradually positively decreasing, on the other hand for the large values of $M<0$ the behavior of deflection angle negatively increasing. But for large value of $q$ and if $M \geq 0$ the behavior of deflection angle is decreasing, on contrary for $M<0$ the behavior of deflection angle gradually negatively increasing.
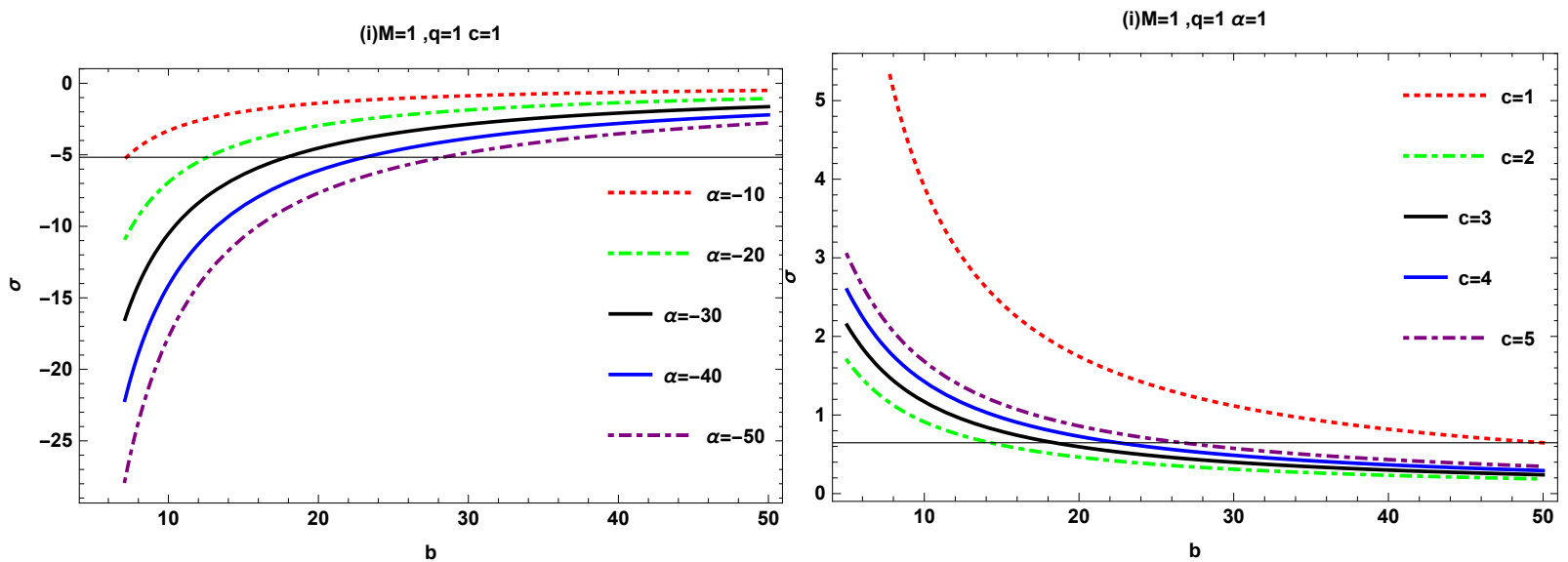

Figure 1: $\alpha$ versus $b$.

- Figure Left indicates the behavior of deflection angle w.r.t $b$ by fixing the value of $\alpha$ and varying $q . c, M$ It is to be observed that for values of $\alpha \geq 0$ the behavior of deflection angle gradually decreasing. For small values of $\alpha$ we noticed that the behavior of deflection angle overlaping. For value $\alpha<0$ the deflection angle gradually negatively increasing.

- Figure Right represents the behavior of deflection angle w.r.t $b$ by varying the $c$ and taking $q, \alpha, M$ fixed. We noticed that for values of $b>5$ and $c \geq 0$ the behavior of deflection angle gradually positively decreasing, on the other hand for the values of $b<5$ the behavior of deflection angle linear. For the values of $c<0$ the behavior of deflection angle is negatively decreasing.

\section{GRAPHICAL ANALYSIS FOR PLASMA MEDIUM}

This section gives us graphical analysis of deflection angle $\alpha$ in the presence of plasma medium. For simplicity we take $C \circ=C$ 

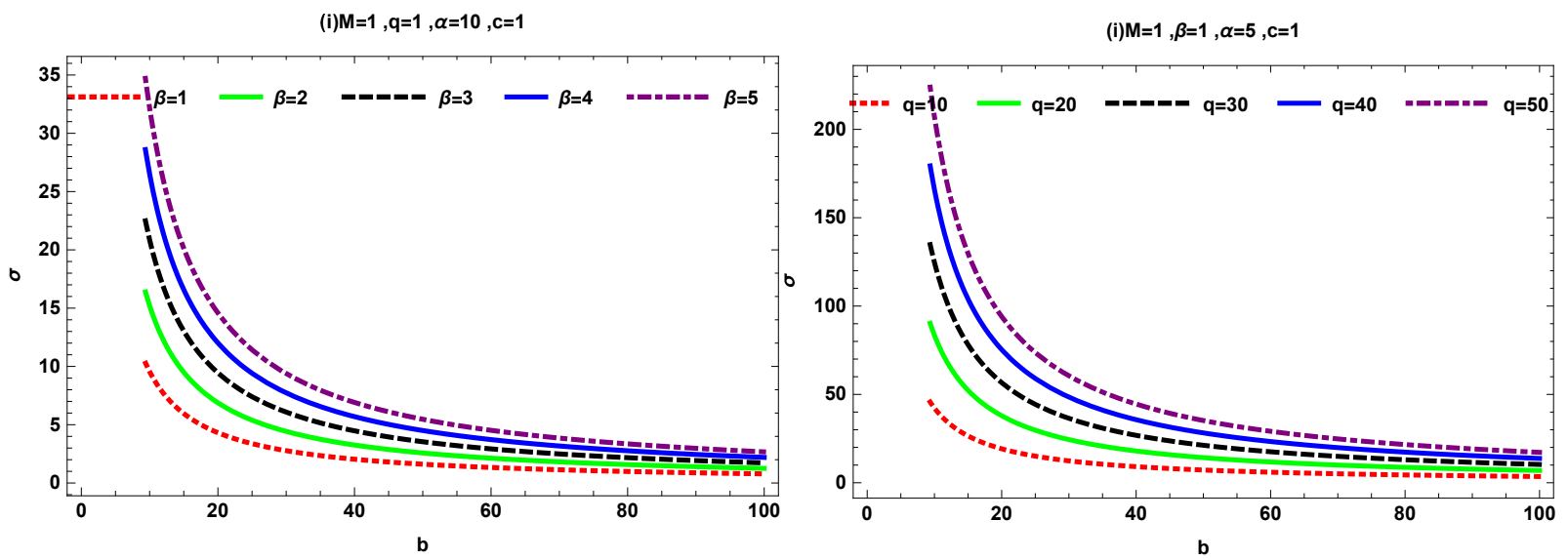

Figure 2: $\tilde{\alpha}$ versus $b$.

- Left plot, shows the behavior of deflection angle w.r.t $b$ by taking the fixed values of $M, q, c, \alpha$ and by varying $\beta$. It is to analyzed that initially the behavior of deflection angle is positively decreasing for $\beta \geq 0$. But for $\beta<0$, the behavior of deflection angle gradually negatively increasing. Furthermore, in the absence of charge and value of $\beta \geq 0$ the behavior of deflection angle positively decreasing.

- Right plot, illustrate the behavior of deflection angle w.r.t $b$ by varying BH charge $q$ and for fixed $M, \alpha, c$ and $\beta$. We examined that for positive values of $q$ the deflection angle gradually positively decreasing. While, for negative values of $q$ the behavior of deflection angle is gradually increasing. For large value of $M, \beta$ and $q>0$ the behavior of deflection angle also gradually decreasing.
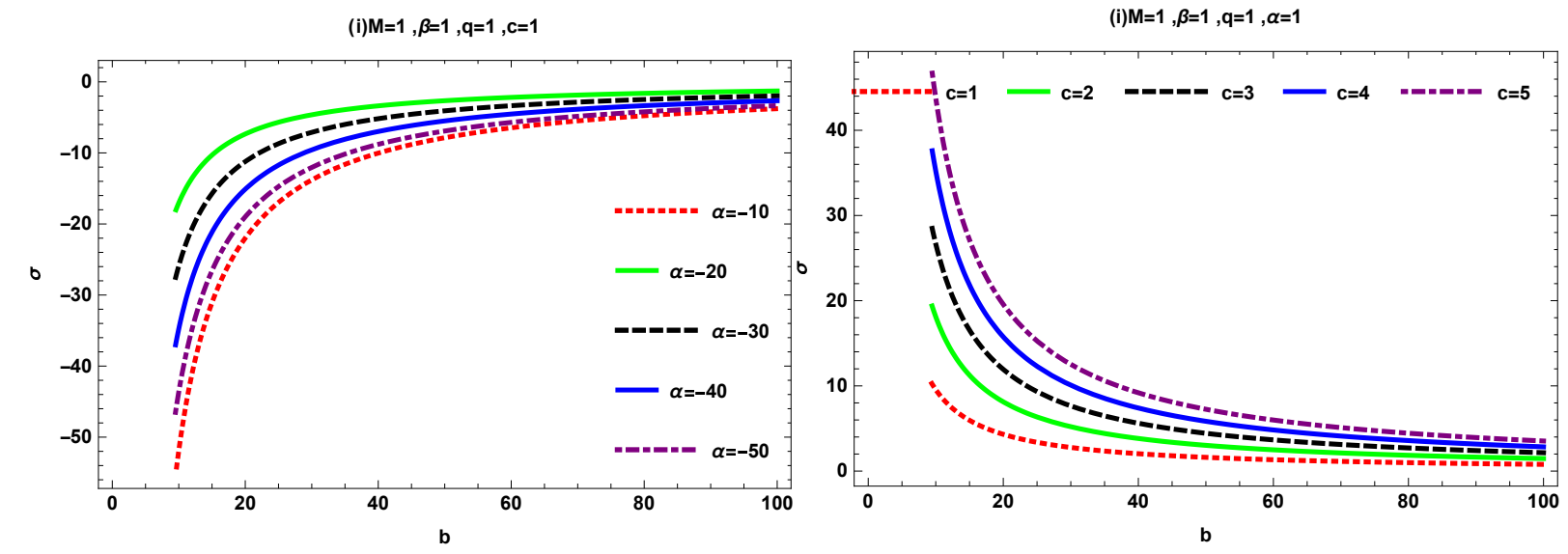

Figure 2: $\tilde{\alpha}$ versus $b$.

- Left plot, examines the behavior of deflection angle w.r.t $b$ by taking the fixed values of $\beta, q, c, M$ and by varying $\alpha$. We noticed that for small values of $\alpha$, and $\beta=1, q=1$ then the deflection angle cannot define the behavior. While, for large values of $\alpha$ the behavior of deflection angle is positively decreasing. For values of $\alpha<$ the behavior of deflection angle gradually increasing.

- Right plot, we analyzed the behavior of deflection angle w.r.t $b$ by varying $c$ and for fixed $M, \alpha, q$ and $\beta$. we see that for $\geq 0$ the deflection angle positively decreasing. For values of $c<0$ deflection angle is negatively increasing. 


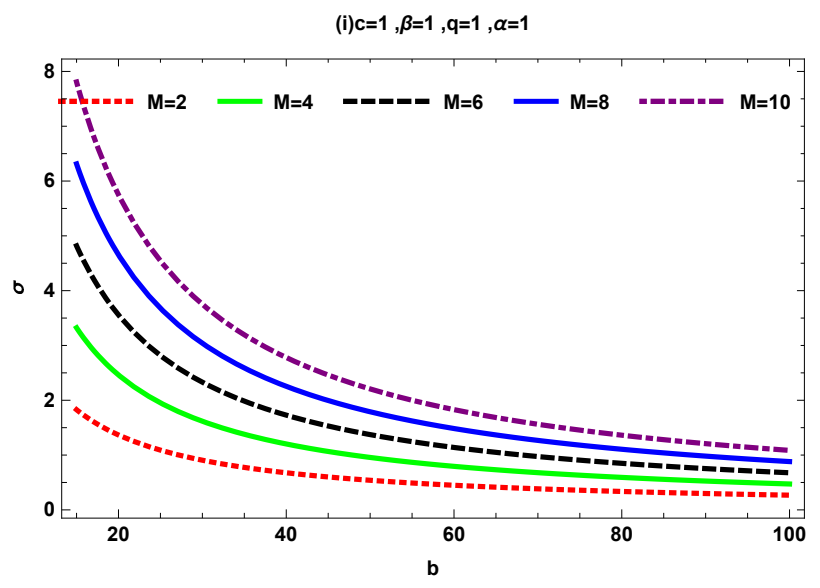

Figure 3: $\tilde{\alpha}$ versus $b$.

- Figure 3, manifest the influence of deflection angle w.r.t $b$ for constant values of $q, \beta, c, \alpha$ and varying $M$. It has been noted that for small range of $b<15$ the behavior of deflection angle not defined. on the other hand for large $b \geq 15$ and for $M \geq 0$ the deflection angle positively decreasing and the behavior of deflection angle is negatively increasing for values of $M<0$.

\section{CONCLUSION}

The present paper is about the investigation of deflection angle by Einstein-non-linear Maxwell-Yukawa $\mathrm{BH}$ in non-plasma as well as plasma medium. In this regard, we study the weak gravitational lensing by using GBT and obtain the deflection angle of photon for Einstein-non-linear Maxwell-Yukawa BH. The obtained deflection angle is given as follows:

$$
\alpha \simeq \frac{4 M}{b}+\frac{C_{0} M \alpha q \pi}{b^{2}}+8 \frac{C_{0} q \alpha}{3 b}-3 \frac{C_{0} q \pi}{b^{2}}-\frac{32 C_{0} M q}{3 b^{3}}+\mathcal{O}\left(q^{2}, M^{2}, C_{0}^{2}\right) .
$$

We examine that by the reduction of some parameters the obtained deflection angle converted into the Schwarzschild deflection angle up to the first order terms. We also discuss the graphical effect of different parameters on deflection angle by Einstein-non-linear Maxwell-Yukawa BH.

We also observed that deflection angle in the presence of plasma medium given by Eq. (4.5). which is;

$$
\begin{array}{r}
\alpha \simeq \frac{4 m}{b}+4 \frac{q \alpha C_{0} M \omega_{e}^{2} \pi}{b^{2} \omega_{\infty}^{2}}+\frac{C_{0} q M \alpha \pi}{b^{2}}+4 \frac{C_{0} q \alpha \omega_{e}^{2}}{b \omega_{\infty}^{2}}+8 / 3 \frac{q C_{0} \alpha}{b}-5 \frac{C_{0} q \omega_{e}^{2} \pi}{b^{2} \omega_{\infty}^{2}}-3 \frac{q C_{0} \pi}{b^{2}}-\frac{416 q C_{0} M \omega_{e}^{2}}{9 b^{3} \omega_{\infty}^{2}} \\
-\frac{32 C_{0} q M}{3 b^{3}}+6 \frac{M \omega_{e}^{2}}{b \omega_{\infty}^{2}}+\mathcal{O}\left(q^{2}, M^{2}, C_{0}^{2}, \omega_{e}^{3}\right)
\end{array}
$$

If the $\frac{\omega_{e}}{\omega_{\infty}}$ then the plasma effect can be removed. Furthermore, we scrutinized the graphical impact of deflection angle on Einstein-non-linear Maxwell-Yukawa BH in plasma medium versus some parameters.

\section{Acknowledgments}

This work was supported by Comisión Nacional de Ciencias y Tecnología of Chile through FONDECYT Grant $N^{\circ}$ 3170035 (A. Ö.).

[1] S. Guillessen et al., Astrophys.692, (2009)1075.

[2] B.P Abbott, LIGO Scientific and virgo collabrationPhys. Rev. Lett. 116(6),(2016)061102.

[3] C. Darwin, proc. Roy.Soc London , 249,(1959)180.

[4] J.P Luminet, Astron. Astrophys. 7 (1979)228; H.C. Ohanian, Am. J. Phys.55,(1987)428.

[5] E.F. Eiro, G.E. Romero, and D.F Torres, Phy. Rev. D66,(2002)024010. 
[6] R. D. Viollier, F. R. Leimgruber and D. Trautmann, Phys. Lett. B297,(1992)132.

[7] N. Bilic and R. D. Viollier, Phys. Lett. B408,(1997)75.

[8] Akhmedov, E.T., Akhmedova, V. and Singleton, D.: Phys. Lett B642(2006)124 Phys. Rev. Lett. 116(6).

[9] M. Barriola and A. Vilenkin, Phys. Rev. Lett. 63,(1989)341.

[10] P. Jetzer, Phys. Rept. 220,(1992)163.

[11] W. Javed, J. Abbas, and A. Övgün, Eur. Phys. J. C 79, no. 8, 694 (2019).

[12] S. vazques and E. Esteban, Nuovo cim.119B,(2004)489.

[13] V.Bozza, Phy.Rev.D66,(2002)103001.

[14] A unifed treatment, Phys. Rev. D97, (2018)124016.

[15] Vieira, H. S., Bezerra, V. B. and Muniz, C.R.: Annals Phys. 350,(2014)4.

[16] V. Morozvan B. Ahmadov and A.Tursunov, Astrophys space sci. 346,(2013)513.

[17] S. Mao, Bpaczynski, Astrophys J374, (1991)37.

[18] M. Barriola and A. Vilenkin, Phys. Rev. Lett. 63, 341 (1989).

[19] E.F. Eiro .G.E Romero. D.F Torres, Phys.Rev. D66(2002)024010.

[20] D.Valls-Gabaud, AIP conf.proc.861,(2006)1163.

[21] K. Jusufi, A. Övgün, J. Saavedra, P. A. Gonzalez and Y. Vasquez, Phys. Rev. D 97, 124024 (2018).

[22] Akhmedova, V., Pilling, T., de Gill, A. and Singleton, D.: Phys. Lett,B666(2008)269.

[23] Parikh, M.K. and Wilczek, F. Phys. Rev. Lett.85,(2000)5042

[24] A.Einstein, Lens-like action of star by the deviation of light in the gravitational field. Science84(1936)506.

[25] G. W. Gibbons and M. C. Werner, Class. Quant. Grav. 25, 235009 (2008).

[26] M. C. Werner, Gen. Rel. Grav. 44, 3047 (2012).

[27] H. Arakida, M. Kasai, Phys.Rev., D85,(2012)023006.

[28] A. Ishihara, Y. Suzuki, T. Ono and H. Asada, Phys. Rev. D 95, no. 4, 044017 (2017).

[29] A. Ishihara, Y. Suzuki, T. Ono, T. Kitamura and H. Asada, Phys. Rev. D 94, no. 8, 084015 (2016).

[30] T. Ono, A. Ishihara and H. Asada, Phys. Rev. D 99, no. 12, 124030 (2019).

[31] X.Er and S. Mao Mon Not Roy Astron soc.437,(2013)2180.

[32] T. Ono, A. Ishihara and H. Asada, Phys. Rev. D 98, no. 4, 044047 (2018).

[33] G. S. Bisnovatyi-Kogan and O. Y. Tsupko, Grav. Cosmol. 20, no. 3, 220 (2014).

[34] O. Y. Tsupko and G. S. Bisnovatyi-Kogan, Phys. Rev. D 87, no. 12, 124009 (2013)

[35] G. Crisnejo, E. Gallo, Phys. Rev. D 97(12), 124016 (2018).

[36] W. Javed, j. Abbas and A. Övgün, Phys. Rev. D 100, no. 4, 044052 (2019).

[37] A. Rogers, Mon. Not. Roy. Astron. Soc. 451, no. 1, 17 (2015).

[38] V.Morozvan B.Ahmadov and A.Tursunov, Astrophys space sci 346,(2013)513.

[39] Nozari, K. and Mehdipour, S.H.: Europhys. Lett.84,(2008)20008.

[40] Sharif, M. and Javed, W.: Eur. Phys. J. C72(2012)1997.

[41] Vieira, H. S., Bezerra, V. B. and Muniz, C.R.: Annals Phys. 350(2014)4.

[42] K. Jusufi and A. Övgün, Phys. Rev. D 97, no. 2, 024042 (2018).

[43] G.W. Gibbons and C. M.Warnick, Phys. Rev. D 79, 064031 (2009).

[44] G.W. Gibbons, C. A. R. Herdeiro, C. M.Warnick and M. C.Werner, Phys. Rev. D 79, 044022 (2009).

[45] G.W. Gibbons and M. Vyska, Class. Quant. Grav. 29, 065016 (2012).

[46] C. Bloomer, arXiv:1111.4998 [math-ph].

[47] G.W. Gibbons, Class. Quant. Grav. 33, no. 2, 025004 (2016).

[48] P. Das, R. Sk and S. Ghosh, Eur. Phys. J. C 77, no. 11, 735 (2017).

[49] I. Sakalli and A. Ovgun, EPL 118, no. 6, 60006 (2017).

[50] K. Jusufi, M. C.Werner, A. Banerjee and A. Övgün, Phys. Rev. D 95, no. 10, 104012 (2017).

[51] T. Ono, A. Ishihara and H. Asada, Phys. Rev. D 96, no. 10, 104037 (2017).

[52] K. Jusufi, I. Sakalli and A. Övgün, Phys. Rev. D 96, no. 2, 024040 (2017).

[53] H. Arakida, Gen. Rel. Grav. 50, no. 5, 48 (2018).

[54] A. Övgün, I. Sakalli and J. Saavedra, JCAP 1810, no. 10, 041 (2018)

[55] A. Övgün, I. Sakalli and J. Saavedra, Annals Phys. 411, 167978 (2019).

[56] A. Övgün, G. Gyulchev and K. Jusufi, Annals Phys. 406, 152 (2019).

[57] A. Övgün, K. Jusufi and I. Sakalli, Annals Phys. 399, 193 (2018).

[58] K. Jusufi and A. Övgün, Phys. Rev. D 97, no. 6, 064030 (2018).

[59] A. Övgün, Phys. Rev. D 98, no. 4, 044033 (2018)

[60] T. Ono, A. Ishihara and H. Asada, Phys. Rev. D 99, no. 12, 124030 (2019).

[61] G. Crisnejo, E. Gallo and A. Rogers, Phys. Rev. D 99, no. 12, 124001 (2019).

[62] Z. Li and T. Zhou, arXiv:1908.05592 [gr-qc].

[63] Z. Li and J. Jia, arXiv:1912.05194 [gr-qc].

[64] Z. Li, G. He and T. Zhou, arXiv:1908.01647 [gr-qc].

[65] A. Övgün, Universe 5, no. 5, 115 (2019).

[66] W. Javed, R. Babar, A. Ovgun, Phys. Rev. D 99, no. 8, 084012 (2019).

[67] W. Javed, A. Hazma and A. Övgün, Preprints 2019, 2019110142 (doi:10.20944/preprints201911.0142.v1)

[68] A. Övgün, Phys. Rev. D 99, no. 10, 104075 (2019).

[69] K. Jusufi and A. Övgün, Int. J. Geom. Meth. Mod. Phys. 16, no. 08, 1950116 (2019). 
[70] G. Crisnejo, E. Gallo, and J. R. Villanueva, Phys. Rev. D 100, no. 4, 044006 (2019).

[71] W. Javed, R. Babar, and A. Övgün, Phys. Rev. D 100, no. 10, 104032 (2019)

[72] K. de Leon and I. Vega, Phys. Rev. D 99, no. 12, 124007 (2019)

[73] Y. Kumaran and A. Ovgün, Chin. Phys. C 44, 025101 (2020).

[74] W. Javed, J. Abbas, Y. Kumaran and A. Övgün, Preprints 2019, 2019110210 (doi: 10.20944/preprints201911.0210.v1).

[75] A. Övgün, I. Sakalli and J. Saavedra, arXiv:1908.04261 [gr-qc].

[76] K.Jusufi, A. Övgün, A. Banerjee, and I. Sakalli, Eur. Phys. J. Plus 134, no. 9, 428 (2019).

[77] K. Jusufi, A. Övgün, and A. Banerjee, Phys. Rev. D 96, no. 8, 084036 (2017).

[78] Z. Li and A. Övgün, arXiv:2001.02074 [gr-qc] (Accepted to publish in PRD).

[79] S. Habib Mazharimousavi and M. Halilsoy, Int. J. Mod. Phys. D 28, no. 09, 1950120 (2019).

[80] M. Abramowitz and stegun, Handbook of Mathematical Function,(United State Department of Commerce, National Institute of Standards and Technology (NBS),1967). 